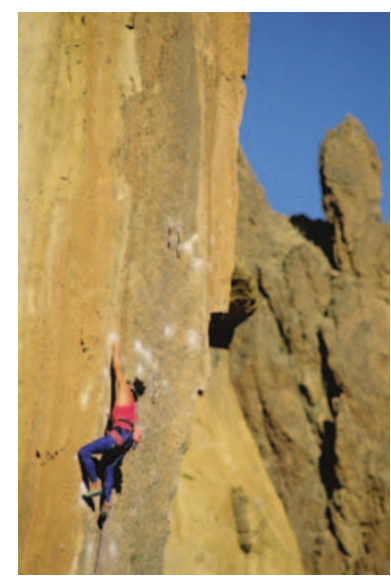

RESEARCH HIGHLIGHTS

URLs

MICROBIAL GENETICS

\title{
Embrace your inhibitions!
}

A thought-provoking new study indicates that inhibiting mutation in the genomes of pathogenic bacteria might be a viable strategy for dealing with the growing problem of antibiotic resistance.

Floyd Romesberg and colleagues used resistance to the synthetic antibiotic ciprofloxacin in Escherichia coli as their model system. Ciprofloxacin interferes with topoisomerases such that they introduce double-strand breaks (DSBs) in the DNA, so it is mutations in genes that encode the topoisomerases (for example, gyrA) that can confer resistance in bacteria. Previous work indicated that, when challenged with antibiotics, bacteria actively induce proteins that promote such mutations.

The SOS response - a DNArepair process that is triggered by the autoproteolytic activity of the gene repressor LexA - was thought to be a key component of the mutation-induction process. The authors used a mouse model of thigh infection to show that LexA was involved: a lexA-mutant form of E. coli (lexA (Ser119Ala)) did not develop resistance to ciprofloxacin, whereas $3 \%$ of the control strain population did so within 72 hours. Repeating these experiments with an antibiotic from a different class - rifampicin - produced qualitatively similar results.

The authors followed up these studies with in vitro experiments that allowed them to compare the number of ciprofloxacin-resistant mutants that arise before and after exposure. From these experiments they estimated that ciprofloxacin induces a 104-fold increase in the rate of evolution of resistance in control strains of E. coli. By contrast, the post-exposure mutation rate was 100-fold lower in the lexA (Ser119Ala) strain, confirming the in vivo finding that LexA derepression was necessary for efficient induction of resistance.

In an attempt to identify the downstream components of the pathway that LexA triggers, Cirz et al. then undertook an elegant series of similar studies of pre- and post-exposure mutation rates for different $E$. coli strains with deletions of various genes in candidate pathways. These experiments provided clearcut evidence that both nucleotide excision repair and recombinational gap-repair pathways are not involved, whereas RecBC-mediated homologous recombination is. Moreover, the authors showed that the effect of deleting any of the three LexA-repressed polymerases (PolII, PolIV and PolV) was equivalent to preventing LexA cleavage: so it seems that LexA functions through derepression of all these enzymes to induce mutations.

The authors suggest that the recombinational DNA-repair pathway might also underlie bacterial responses to other antibiotics, and indeed other cellular challenges. Such a neat system of mutational feedback, which is designed to restore the evolutionary status quo, certainly has an intuitive appeal. Moreover, if true, it would also have huge implications for our understanding of, and therapeutic approach to, antibiotic resistance.

Nick Campbell, NPG Executive Editor, Heredity

(S) References and links ORIGINAL RESEARCH PAPER Cirz, R. et al. ORIGINAL RESEARCH PAPER Ciz, R. et al.
Inhibition of mutation and combating the evolution of antibiotic resistance. PLOS Biol. 3, e176 (2005) FURTHER READING Hughes, D. Exploiting genomics, genetics and chemistry to combat antibiotic resistance. Nature Rev. Genet. 4 , 432-441 (2003)

WEB SITE

Floyd Romesberg's laboratory: http://www. scripps.edu/chem/romesberg 